\title{
Cytokine Networks Regulating Inflammation and Immune Defense in the Oral Cavity
}

\author{
Franco Cavalla • Ana Claudia Araujo-Pires • \\ Claudia C. Biguetti • Gustavo P. Garlet
}

Published online: 27 March 2014

(C) Springer International Publishing AG 2014

\begin{abstract}
The host/pathogen interaction in infectious oral diseases is characterized by complex and precisely orchestrated host response mechanisms aimed to protect the host against the microbial challenge with minimal collateral damage to host cells and tissues. Central to the host response in this battlefront is the expression of cytokines. The resulting cytokine networks, which ultimately regulate the host response at multiple levels, thereby determine the clinical outcome of the disease and explain most of its defining features from a mechanistic viewpoint. This review intends to present a structured view of the intricate cytokine networks that regulate inflammation and immune defense in the oral cavity, guiding the reader throughout the evolving paradigms that describe how the simultaneous action of multiple cytokines shapes the nature of the immune response to oral infection.
\end{abstract}

Keywords Cytokine networks $\cdot$ Inflammation $\cdot$ Immune defense $\cdot$ Oral cavity $\cdot$ Immunology

\section{Introduction}

Periodontal and periapical diseases are the most common forms of destructive infectious/inflammatory diseases of the

F. Cavalla $(\bowtie) \cdot$ A. C. Araujo-Pires $(\bowtie) \cdot$ C. C. Biguetti $(\bowtie) \cdot$ G. P. Garlet $(\bowtie)$

Osteoimmunology Laboratory, Department of Biological Sciences, School of Dentistry of Bauru, University of Sao Paulo (FOB/USP), Al. Otávio Pinheiro Brisola 9-75, 17010-901, SP São Paulo, Brazil e-mail: francocavalla@usp.br

e-mail: anaclaudia.araujo@usp.br

e-mail: biguetti@usp.br

e-mail: garletgp@usp.br

F. Cavalla

Periodontal Biology Laboratory, Faculty of Dentistry, University of

Chile, Sergio Livingstone Pohlhammer 943, Santiago, Chile tooth-supporting tissues, and represent the most prevalent form of bone pathology. Periodontitis is initiated by bacteria harbored in the tooth-attached biofilm invading the surrounding epithelial and connective periodontal tissues, triggering a host immune/inflammatory response and the subsequent lesion development. Similarly, lesions of endodontic origin initiate as an immune/inflammatory response to a bacterial insult of the dental pulp, which can lead to necrosis, allowing the spread of the infection front to the periapical region and the consequent lesion formation. Although both conditions share a quite common etiology, it is noteworthy that based on bacterial infection alone, it is not possible to explain these complex pathological processes [1, 2, 3••]. Indeed, while any bacteria can essentially trigger a host response, the current paradigm of periodontal and periapical disease etiology states that specific bacteria (gram negative anaerobic rods) initiate and chronically sustain the host immune/inflammatory response, the nature and extent of which are ultimately responsible for the degree of irreversible tissue destruction and disease severity [4].

In this scenario, the interplay between the challenging microorganisms and resident and inflammatory host cells is thought to determine the disease outcome. Theoretically, if the host response is efficient in keeping the microorganisms spatially confined and in limited number, the tissue homeostasis will then be preserved. Conversely, if the host response is incapable of counteracting the bacterial challenge (where the presence of specific pathogens presenting virulence factors that allow tissue invasion or interfere with host's defense mechanisms is supposed to be a critical event; as well the rupture of physical barriers that limit bacterial infiltration such as enamel/dentin destruction), the result will be conversion to chronic inflammation, with destruction of the surrounding tissues as a consequence $[5,6]$.

The initial host cells' innate response involves the recognition of microbial components (i.e., LPS, bacterial DNA, 
diacyl lipopeptides, peptidoglycan, etc.) as "danger signals" by pattern recognition receptors, such as the toll-like receptors (TLRs) [7]. TLRs are expressed by both resident cells and leukocytes in the periodontal/periapical environment, and upon their activation, an intracellular signaling cascade is stimulated, leading to activation of the transcription factors that mediate the cellular response and the subsequent production of inflammatory mediators, such as cytokines [8].

Cytokines exert their functions through binding to specific cellular receptors, therefore both cytokine and receptor expression can determine the nature of an individual cell response, and in a broader context, can impact on the intensity and duration of the inflammatory response, and ultimately the clinical outcome of periodontal/periapical disease. Over the last few decades we have accumulated a broad but somewhat superficial knowledge on how individual cytokines contribute to the development of these diseases. Recently, the application of new technology and analytical tools have revealed the multitude and complexity of cytokines, especially regarding the selective synthesis and secretion of effector cytokines by specific $\mathrm{CD} 4{ }^{+}$T-lymphocyte subsets, and their participation in complex regulatory networks.

Numerous studies have intended to characterize the host response to infection in periodontal/periapical diseases; providing valuable information on the interactions between the bacterial biofilm and the responding host cells [9-11]. With the progress of our knowledge from purely descriptive observations towards more mechanistic findings, we are beginning to get a reasonable glimpse of the whole picture. In this context, the present review intends to present a chronological perspective on the development of the current hypothesis on the etiology of periodontal and periapical diseases, focusing on the specific contribution of cytokines and cytokine networks, and on how our increasing knowledge and insight into the immune processes is producing a more complete account of the extremely complex phenomena that characterizes these destructive diseases (Table 1, Figure 1).

\section{Pro-inflammatory and Anti-inflammatory Cytokines in the Host Innate Immune Response}

The initial investigations of immune response to bacterial infection in periodontal/periapical diseases demonstrated that both resident and infiltrating cells were able to recognize oral bacteria (and its antigens), and respond to them in a proinflammatory manner. In the case of periodontitis, the gingival epithelium is the first line of defense to infection and is routinely challenged by oral bacteria, constitutively secreting cytokines that attract a moderated but constant influx of immune cells (predominantly neutrophils), which arrest the bacterial growth and prevent infiltration [4, 12]. Epithelial cells respond to the periodontal infection by up-regulating pro- inflammatory cytokine/chemokine expression, thereby providing the stimuli for the leukocyte recruitment that will lead to the latter disease stages [13].

After the initial response of epithelial cells to the microbes, the permeability of the junctional epithelium is increased by the inflammatory mediators, and allows the contact of bacteria (and/or their products) with gingival connective tissue, where most of the host/microbe interaction events occur. Indeed, gingival fibroblasts (GF), the predominant cell type in gingival connective tissue, possess the capacity to respond to infection with the secretion of pro-inflammatory mediators that trigger, and to some extent sustain, the initial steps of the immune response [14].

In the periapical lesion, the spread of the infection front across the root apex/periodontium results in the direct contact of the infecting microbes with the periodontal ligament fibroblasts, that although not routinely exposed to bacterial challenge, also have the capability to respond to infection with cytokine production, participating in the initial recruitment phase of the host response similar to GFs [3••]. It is important to note that leukocytes can also play a role in the initial steps of host response to oral infections, since neutrophils and resident macrophages can be found in clinically healthy connective periodontal, pulpal and periapical tissues.

Within the pro-inflammatory mediators thought to coordinate the inflammatory cell migration in periodontal/periapical tissues, TNF- $\alpha$, IL-1, and IL- 8 have been extensively investigated. IL-1 and TNF- $\alpha$ share several pro-inflammatory properties, paramount among them the capacity to activate endothelial cells to provide the signals required for leukocyte diapedesis $[3 \bullet \bullet]$. Indeed, these pro-inflammatory cytokines induce the production of chemokines. These selectively recruit leukocytes from the peripheral circulation to the site of infection via specific ligand-receptor interaction that ultimately triggers integrin-dependent adhesion; cytoskeletal rearrangement to facilitate extravasation and migration, as well as the binding and detachment of cells from their substrate [5]. In the cell migration framework, IL-8 (CXCL8) is the prototypical and firstly identified member of the chemokine family [15]; specifically acting in the chemoattraction of neutrophils, which form a sub-epithelial barrier that exerts a potent microbicidal action by means of its secretory functions (reactive oxygen species and bactericidal proteins), and acts as a unified phagocytic apparatus [16]. IL-8 production can be directly induced by TLR stimulation, or indirectly via TNF- $\alpha$ and/or IL-1 [17]. In periodontitis patients, IL- 8 level is significantly increased compared with gingivitis-affected and healthy subjects [18], and seems to correlate with the periodontal status of the patients before and after treatment $[19,20]$. While the early neutrophil migration is under the control of IL-8, the developing chronic inflammatory response also involves the migration of macrophages, where CCL2 (MCP-1) appears as a master regulator of monocyte 


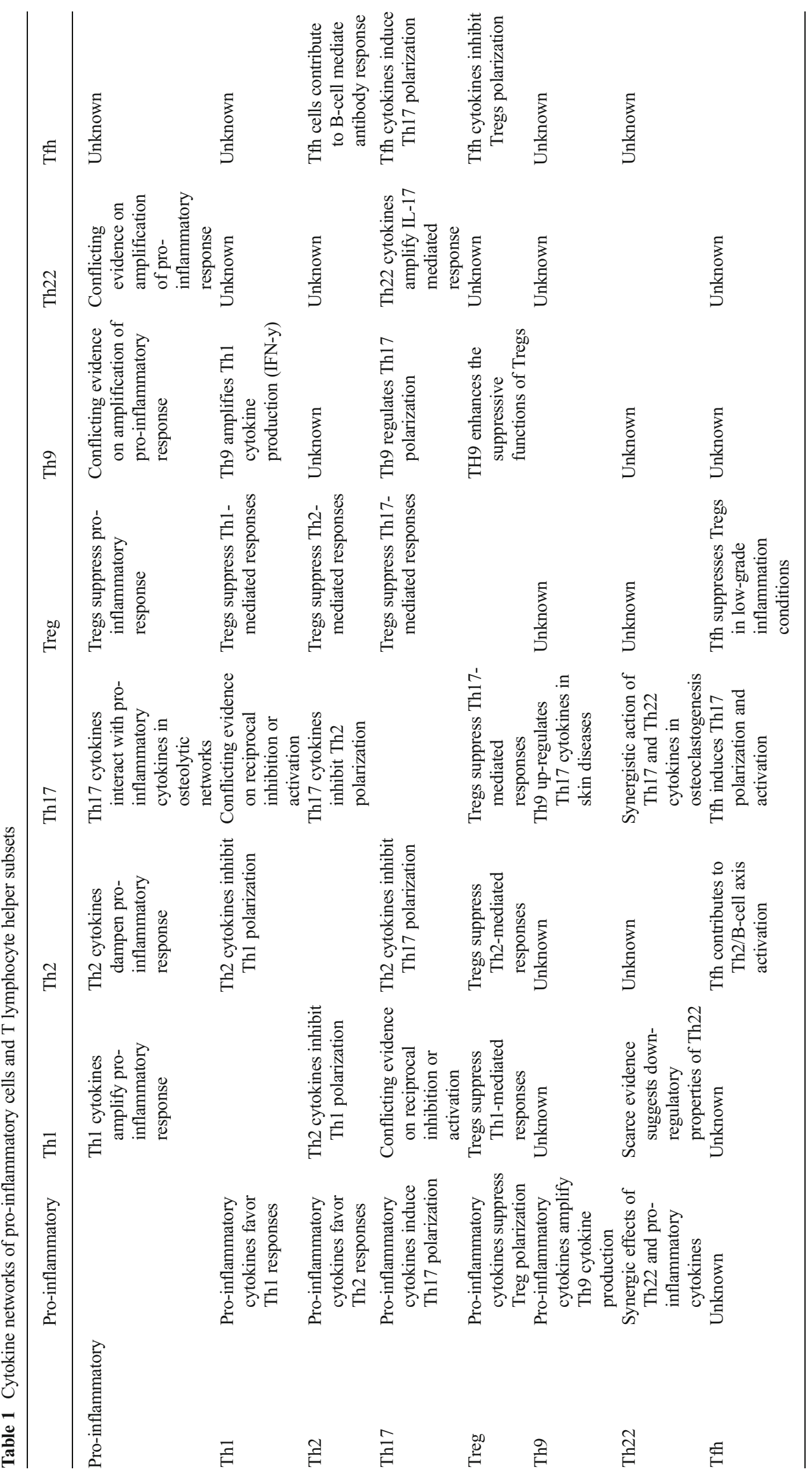




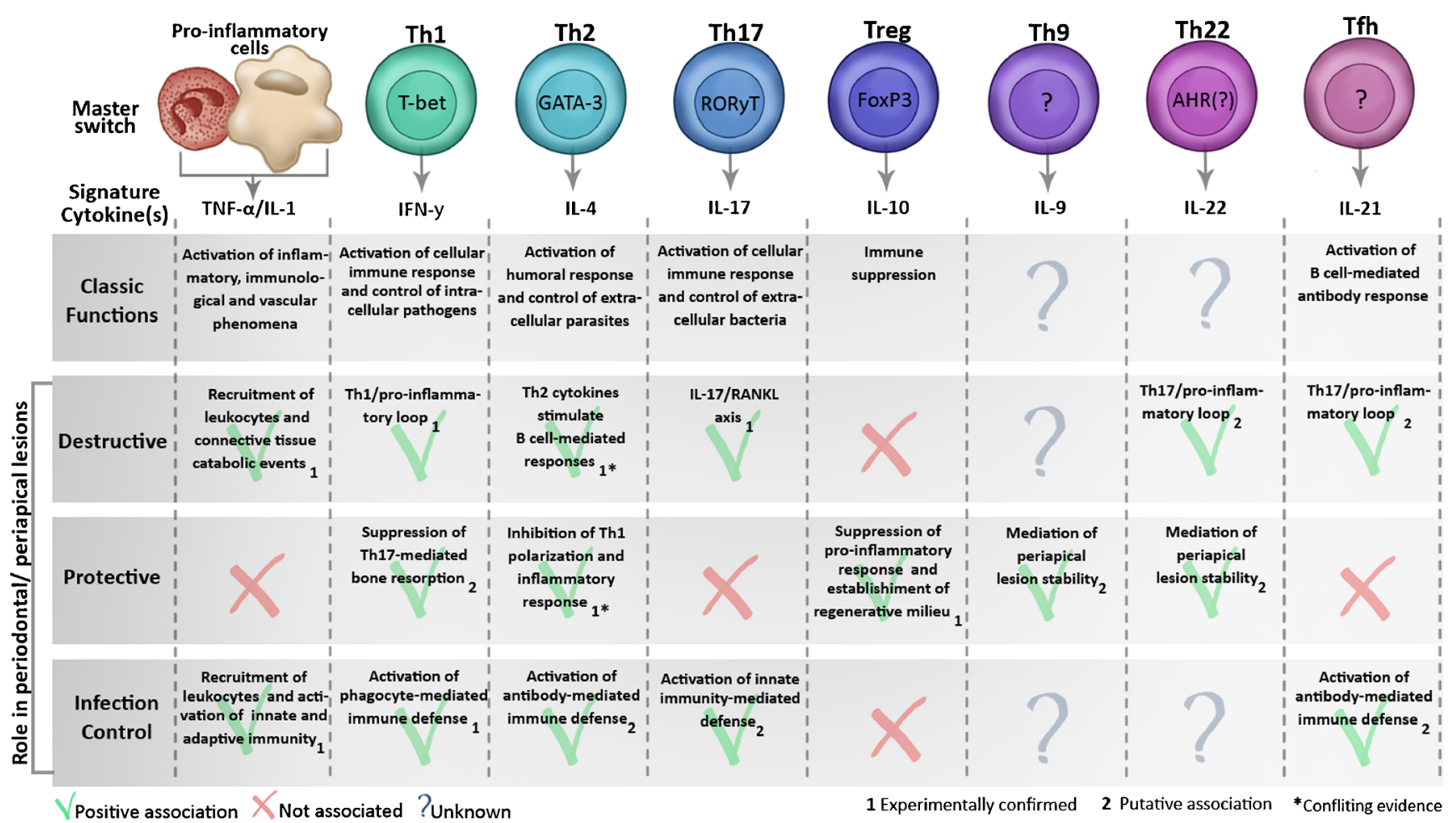

Fig. 1 Defining features of pro-inflammatory and T lymphocyte helper cell subsets in periodontal and periapical lesions. Pro-inflammatory and T lymphocyte helper cell subsets present characteristic features (such as master switches, signature cytokines and classic functions) that define such cell subset in phenotypic and functional terms. When these cells are considered in the context of periodontal and periapical lesions, they have putative roles in the tissue destructive/protective responses, as well in the control of infection mobilization [21, 22]. The level of CCL2 is increased in the saliva and gingival crevicular fluid (GCF) of periodontitis patients, and correlates with periodontitis severity [23]. It has also been reported that CCL2 levels are increased in the GCF and serum of periodontitis-affected smokers compared with non-smoking patients, suggesting that this chemokine could be involved in the worsening of the periodontal condition characteristic of the former [24]. Similar to periodontitis, IL-8 and CCL2 can be described as coordinating the respective migration of neutrophils and macrophages during the development of periapical lesions $[25,26]$.

Once in the periodontal/periapical tissues, neutrophils and macrophages seem to act along epithelial and connective tissue cells towards the maintenance and amplification of the inflammatory/immune response. Interestingly, both neutrophils and macrophages can be a significant source of TNF- $\alpha$ and IL-1, generating a positive loop towards response amplification [27].

Taken together, the pro-inflammatory cytokines can account for a significant extent of the tissue destruction associated with periodontal/periapical diseases, since they induce matrix metalloproteinases (MMPs) and receptor activators of nuclear factor kappa-B ligand (RANKL), the main mediators of soft and hard tissue destruction, respectively. Indeed, both soft connective tissue and bone integrity depend on the balance between resorption/deposition. The overexpression of pro-inflammatory cytokines in periodontal and periapical chronic inflammation interferes with the physiologic equilibrium, leading to pathologic tissue loss [2].

Even though pro-inflammatory cytokines have been classically related to destructive events, they also exert pivotal roles in the control of infection. Surprisingly, only recently has the "protective" nature of cytokines against infection been systematically investigated. TNF- $\alpha$ has a central role in the control of periodontal/periapical infections, and its deficiency generates a severe pathogen clearance impairment, increasing the systemic acute phase response. Indeed, TNF- $\alpha$ is a prototypical phagocyte activator, mediating both myeloperoxidase (MPO) and inducible nitric oxide synthase (iNOS) expression, respectively the major antimicrobial effectors of neutrophils and macrophages [28]. Accordingly, the lack of iNOS results in an increased periapical abscess formation following endodontic infection [29].

While the pro-inflammatory response reveals a dual nature (destructive while protective against infection), it is also important to remember that there are other cytokines involved in the overall cytokine network to prevent/attenuate the side effects of an exacerbated response. In this context, it is worthwhile to highlight the functions of IL-10, that antagonizes proinflammatory cytokine secretion and signaling, downregulates MMP activity (directly and indirectly via tissue inhibitors of MMPs), and suppresses RANKL-mediated 
osteoclastogenesis (via osteoprotegerin) [30, 31]. These properties point to IL-10 as a "protective" mediator in periodontal/ periapical lesions, although we must bear in mind that the protective/destructive dichotomy is a partial and oversimplified interpretation (albeit instructive) of a more complex phenomenon, given the dual role of host response previously discussed [2].

After the initial innate response characterized by the balance between pro-inflammatory vs. anti-inflammatory cytokines, the selective migration of specific T lymphocyte subsets drives the transition to the adaptive immune response; where $\mathrm{T}$ helper lymphocytes (Th) play a critical role in orchestrating the response. As described in the sequence, the discovery of Th subpopulations dramatically changed the understanding of cytokine networks beyond the proinflammatory vs. anti-inflammatory framework.

\section{The Th1/Th2 Balance Paradigm}

The recognition in the late 1980s of two distinct (Th1 and Th2) clonal linages of Th lymphocytes, defined by unique cytokine secretory patterns, was a cornerstone in the understanding of immunological responses and the development of the current paradigms of immune/inflammatory disease etiology $[32,33]$. Th1 cells mediate immune responses against intracellular pathogens and are particularly important in infection control. A dendritic cell-derived cytokine, IL-12, is the principal cytokine responsible for Th1 lineage commitment and differentiation, which involves the activation of the key transcription factor T-bet [34]. The prototypic cytokine product of Th1 lymphocytes is IFN- $\gamma$, involved in the activation of macrophage microbicidal functions [35, 36]. In parallel with Th1 cell discovery, their opposing mates Th2 were also unraveled. Th2 cells mediate the host defense against extracellular parasites and secrete a wide array of cytokines, including IL-4, IL-5, IL-9, IL-13, and IL-25. IL-4 is the main positive feedback signal for Th2 lineage commitment via induction of the GATA-3 transcription factor [37]. The selective polarization to Th1 or Th2 lineage is dependent upon the cytokine milieu in which the antigens are presented to the naïve $\mathrm{T} \mathrm{CD} 4^{+}$cells, in that the polarization is a mutually exclusive event that also generates subsequent positive feedback to the respective lineage polarization [38].

The Th1/Th2 dichotomy paradigm was quickly replaced by a more realistic Th1/Th2 balance hypothesis, where the distinctive features of immune response could be explained by the predominance of one clonal Th subset over the other, comprising a theoretical framework to investigate $\mathrm{T}$ cellmediated immunological responses and related diseases, including periodontal/periapical diseases. The Th1 predominance hypothesis in periodontal/periapical diseases was supported by experimental evidence of the overexpression of
IFN- $\gamma$ in gingival mononuclear cells isolated from periodontitis patients [39], and by the quantitative dominance of IFN- $\gamma$ over IL-4 in the lesional tissue/fluid of periodontal/periapical disease patients $[21,40]$. The inner rationale for the Th1 predominance hypothesis of periodontal destruction was that the exacerbated activation of neutrophils and macrophages by Th1 cytokines interfered with, and amplified, the proinflammatory activity of phagocytes and even of resident fibroblasts, and ultimately increased local levels of MMPs and RANKL, leading to augmented soft tissue and bone destruction [41-43]. While potentially destructive, IFN- $\gamma$ mediated Th1 responses are essential to avoid pathogen dissemination. Indeed, IFN-KO mice challenged by A. actinomycetemcomitans oral infection presented a less severe periodontitis phenotype, but on the other hand suffered a widespread (and often lethal) systemic infection, associated with lower MPO and iNOS production [44].

Conversely, other studies supported the Th2 predominance hypothesis in periodontal/periapical diseases, based on the fact that Th lymphocytes isolated from inflamed gingival tissues predominantly produced IL-4 over IFN- $\gamma$, after nonantigen specific stimulation [45]. Also, the initial descriptions of established periodontal lesions as a "B-cell lesion" [46] were used to support the possible Th2 role in periodontitis progression in the view of the $\mathrm{Th} 2 / \mathrm{B}$-cell cooperative axis. In this framework, the Th2-stimulated local production of antibodies would be a protective factor, albeit not fully supported by experimental evidence. However, some authors consider that Th2 polarization may indicate an impaired adaptive immune response, in which Th2 cytokines inhibit Th1 polarization (and consequently the phagocytic immune mechanisms), due to inherent host characteristics, or by evasion strategies mediated by virulence factors of periodontopathic bacteria [47-49]. When the pro-inflammatory versus antiinflammatory and Th1 vs. Th2 frameworks are considered in a global context, evidence points to a cooperative role of Th1 and pro-inflammatory cytokines in the amplification of tissue destructive events in gingival/periapical tissues; while Th2 mediators seem to cooperate with anti-inflammatory cytokines, given the similarity between the properties of IL-10 and IL-4 [50, 51].

Interestingly, conflicting data were readily available in the literature to support either the Th1 or Th2 predominance hypotheses. The heterogeneity of experimental design, disease definitions, time of sample collection, stimulation protocols, and analytical methods used in the different studies could explain some of these differences in outcome [51]. Additionally, the discovery and recognition of new $\mathrm{T}$ helper cell lineages overcame the traditional, and quite limited, Th1/ Th2 balance hypothesis that restrained the full understanding of the cytokine networks operating in periodontal/periapical lesion development [50]. It will be discussed below how the expansion of the Th family has led to a new level of 
complexity of cytokine networks, where multiple sets of $\mathrm{T}$ cells can reciprocally inhibit or stimulate the functions of another Th set and by doing so, orchestrate the outcome of adaptive immune responses.

\section{Th17 and Treg}

When the relatively simple Th1/Th2 dichotomy and balance theories proved insufficient to fully explain the pathogenesis of a series of immune response-related conditions, the identification of additional Th subsets brought a new perspective, and additional complexity, to the evolving cytokine networks. In 2003, it was demonstrated that IL-23 could drive a population of T cells to express the transcription factor ROR $\gamma \mathrm{T}$ and to secrete IL-17 $[52,53]$. These cells were later named Th17 and are able to secrete IL-17, IL-21 and IL-22, with IL-17 being the cytokine which characterizes the effector functions. IL-17 was found to be largely expressed in inflamed periodontal/periapical lesions $[43,54,55]$. Our group reported overexpression of IL-17, IL-23, IL-6, TGF- $\beta$ and IL- $1 \beta$ in the gingival tissues of periodontitis patients compared to healthy controls, as well as augmented levels of both IL-17 and RANKL in alveolar bone from periodontitis-affected subjects [56]. These results provided evidence that the inflammatory context characteristic of periodontal disease (i.e., IL-1 $\beta$, IL-6, and TGF- $\beta$ ) could drive the polarization of the Th17 subset in human periodontitis. Th17 cells are supposed to contribute to the development of periodontal/periapical lesions directly upregulating MMPs, and indirectly by an inflammation amplification loop stimulating the secretion of the classic proinflammatory cytokines by other cell types $[57,58]$.

Interestingly, shortly after the discovery of Th17 cells, studies characterized their interplay with the previously known Th1 and Th2 subsets, since both Th1 and Th2 cytokines were recognized to antagonize IL-17 secretion and Th17 polarization [59]. Most notably, it was demonstrated that IFN- $\gamma$ neutralization worsened the tissue damage in a delayed-type hypersensitivity model, providing the first evidence that the Th1 subset could arrest the tissue destruction mediated by IL-17, reversing the classical pro-inflammatory and pro-destructive stereotype assigned to Th1 lymphocytes $[52,60]$. When a possible Th1/Th17 interplay is considered in the periodontal context, it was observed that progressive periodontal sites demonstrated overexpression of RANKL, IL-1 $\beta$, IL-17 and IFN- $\gamma$ compared with inactive sites, and positive correlations exist between RANKL/IL-17, ROR $\gamma$ T/RANKL, and ROR $\gamma \mathrm{T} / \mathrm{IL}-17$. These studies indicate the putative involvement of the Th17/IL-17/RANKL axis in periodontitis progression [61, 62]. Interestingly, unpublished data from our group derived from molecular analysis of active periapical lesions demonstrate an inverse correlation between IFN- $\gamma$ and IL-17 levels, suggesting that Th1 and Th17 pathways may operate independently (and not cooperatively) in the evolution of periapical lesions.

Switching to the immunosuppressive front, a new Th lineage with broad immunosuppressive properties was characterized, including the inhibition of Th1, Th2 and Th17 polarization [63]. This subset was named T regulatory (Tregs) and it was defined by the expression of the phenotypic markers CTLA-4, IL-10, TGF- $\beta$, GITR, CD103, and CD45RO; and Foxp3 was shown to be the master transcriptional regulator for Tregs differentiation [38]. Tregs were associated with high expression of the regulatory cytokines IL-10 and TGF- $\beta$ in inflamed periodontal tissues [31], and their presence was associated with decreased disease severity [64]. Conversely, some studies have reported an overexpression of Foxp3, Tbet, RANKL, IL-17, IL- $1 \beta$, and IFN- $\gamma$ in human active periodontal lesions, compared with inactive ones [61, 62]. Other studies have shown that IL-10-expressing cells outnumber the pro-inflammatory cytokine-expressing cells in human periapical lesions [65], and that the proportion of Foxp $3^{+}$cells are more prevalent in periapical granulomas and less prevalent in residual radicular cysts [66]. While data from human samples is conflicting regarding the role of Tregs in periodontal/ periapical lesion pathogenesis, experimental data links the presence of Tregs with the attenuation of disease progression rate, such findings being confirmed in a cause-effect manner by disabling Tregs with anti-GITR antibodies [64]. Accordingly, in a recent report we demonstrated that the selective recruitment of Tregs with a controlled release system of the chemokine CCL22 (a known Treg chemoattractant) is effective in reducing the clinical measures of inflammation and bone loss in canine/murine models of periodontitis. Most interestingly, while Tregs have been associated with the impairment of protective immunity in certain diseases, the Tregs local enrichment treatment did not increase the bacterial load in gingival tissues, and did not raise the levels of serum inflammation markers [67••]. This new approach to the periodontal therapy by immune modulation is an excellent example of how immune research could translate to the clinical setting in the near future.

\section{Th9, Th22 and T Follicular Helper}

Even more recently, Th9, Th22 cells, and T follicular helper subsets (Tfh) were discovered and shown to interact with the previously known Th subpopulations in the modulation of inflammatory/immune responses [68].

Th9 cells characteristically produce IL-9, initially designated as a Th2 cytokine, which exerts pro-inflammatory or antiinflammatory activities by modulating Tregs and/or Th17 cell development and function [69]. Th9 cells produce and secrete TNF- $\alpha$ and are involved in pro-inflammatory amplifying loops in many skin diseases [70]. Th22 cells produce IL-22, 
which can exert pro-inflammatory effects by a synergistic action with classic pro-inflammatory mediators such as TNF- $\alpha$ and IL-17 [71, 72]. IL-22 can also directly upregulate RANKL expression and therefore induce osteoclastogenesis [73]. We showed that both Th9 and Th22 cytokines are expressed in human and experimental periapical lesions, where they supposedly contribute to the lesion stability [74••]. However, no mention of the role of these cytokines in periodontal disease can be found in the literature.

Finally, it is important to mention $\mathrm{Tfh}, \mathrm{a} \mathrm{CD} 4^{+} \mathrm{T}$ cell subset found in the B-cell follicles of secondary (and feasibly tertiary) lymphoid organs [75], described as a major contributor to B cell-mediated antibody responses and an important source of IL-21 [76]. IL-21 is a pleiotropic cytokine highly expressed in gingival biopsies of chronic periodontitis [77, 78], and it has been implicated in osteoclastogenesis and bone resorption [79], as well as in the development of Th17 cells [80]. It has also been implicated in the inhibition of Tregs polarization in low-grade chronic inflammatory disorders, such as obesity [81]. Nevertheless, no mentions regarding the possible role of Tfh in either periapical lesions or periodontal disease can be found in the literature so far. Indeed, due to the relative novelty surrounding Th9, Th22, and Tfh subsets, along with the lack of specific studies focused on their patterns of expression (and possible role) in periodontal/periapical lesions pathogenesis, it is not possible to make any definitive conclusion regarding their individual and collective role in the overall cytokine network.

\section{Cytokine Networks: from Classic T-helper Prototypes to the T-cell Plasticity Concept}

Conventionally, the differentiation of naive $\mathrm{CD} 4^{+} \mathrm{T}$ cells into subsets has been considered as an irreversible event, defined by the expression of selective signature cytokines and a single master transcription factor [38]. However, with the discovery of new Th subsets our understanding of the fate of T-cell identities has changed. It has been recently established that the same cytokines can be expressed by more than one polarized Th subset, and that they can also change their phenotype, characterizing a phenomenon described as plasticity $[82$, $83 \bullet \cdot$. As an example, IL-10 was once thought to be a Th2 exclusive cytokine, but it is now clear that IL-10 can be produced by multiple Th subsets, such as Th1, Th2, Tregs, and Th17 cells $[83 \cdot \bullet, 84]$. Similarly, IL-9 is preferentially produced by the Th9 subset [85], but it can also can be produced by Th2, Th17, and Tregs $[69,86]$. Another interesting plastic characteristic of Th cells is the recently discovered possibility of simultaneous expression of the Th17 and Tregs transcription factors (ROR $\gamma \mathrm{T}$ and Foxp3) in the same cell [87]. There are many other examples of Th plastic features and the complexity of these findings calls into question whether Th cell subsets are more appropriately viewed as a "work in progress", rather than a terminally differentiated cell [83••]. Nevertheless, it is noteworthy that the majority of the experimental data regarding Th cell differentiation and plasticity is derived from in vitro studies employing extreme (and possibly artificial) polarizing stimuli to obtain homogenous populations [88], so extrapolations to the clinical setting must be made carefully.

With the perspective of the plastic capabilities of Th lymphocytes in mind, it is possible to hypothesize that the controversies that regularly arise, regarding the functions of all $\mathrm{Th}$ subsets in the pathogenesis of periodontal/periapical diseases could merely be the expression of the continuous metamorphosis of the Th subpopulations along disease stages, possibly as an adaptive reflex to the changing environmental conditions and immunological necessities, in the battlefront of periodontal infection. More powerful analytical tools will be required in order to comprehensibly reappraise our current data and understandings in light of this new viewpoint.

\section{Concluding Remarks}

Currently, great efforts are being made to integrate the overwhelming amount of new data regarding cytokine networks and their involvement in the overall regulation of inflammatory immune responses to periodontal/periapical infection. The discovery and characterization of Th subsets provided the conceptual framework to evaluate complicated data, helping in the development of explanatory models for the intricate immunological process underlying the pathogenesis of periodontal/periapical diseases. As ingeniously portrayed by John Conway in his famous cellular automaton "Game of Life", complex systems could emerge from a limited set of simple rules, and the rules are often impossible to infer from the observation of the working system [89]; the focus of our efforts should therefore be to unveil the underlying rules that govern the biological systems, and not to get caught in the trap of using advanced technology simply to further describe these complex phenomena. For the last 25 years, the Th1/Th2 and the Th17/Tregs paradigms have provided researchers an invaluable intellectual framework to interpret experimental data. With the refinement of research techniques our apparently solid models are changing faster than ever before, and the scope of our understanding should expand accordingly. In trying to keep up with this growing knowledge we must apply the lessons learned and integrate the new concepts and ideas emerging from research data in more complex and holistic models, understanding our current notions and paradigms as flexible ones. The unveiling of Th plasticity opens a new opportunity for revisiting our ideas of cytokine networks in immunological processes; instead of rejecting the fresh notions and attempting to protect the established paradigms, we 
should embrace them and be willing to reinterpret all our accumulated data under this new light.

\section{Compliance with Ethics Guidelines}

Conflict of Interest Dr. Franco Cavalla, Dr. Ana Claudia Araujo-Pires, Dr. Claudia C. Biguetti, and Dr. Gustavo P. Garlet each declare no potential conflicts of interest relevant to this article.

Human and Animal Rights and Informed Consent This article does not contain any studies with human or animal subjects performed by any of the authors.

\section{References}

Papers of particular interest, published recently, have been highlighted as:

•• Of major importance

1. Graves D. Cytokines that promote periodontal tissue destruction. J Periodontol. 2008;79(8 Suppl):1585-91.

2. Garlet GP. Destructive and protective roles of cytokines in periodontitis: a re-appraisal from host defense and tissue destruction viewpoints. J Dent Res. 2010;89(12):1349-63.

3.• Graves DT, Oates T, Garlet GP. Review of osteoimmunology and the host response in endodontic and periodontal lesions. J Oral Microbiol, 2011. 3. The article reviews the role of cytokines and chemokines in the development of osteolytic periodontal/periapical lesions.

4. Kornman KS, Page RC, Tonetti MS. The host response to the microbial challenge in periodontitis: assembling the players. Periodontol 2000. 1997;14:33-53.

5. Silva TA et al. Chemokines in oral inflammatory diseases: apical periodontitis and periodontal disease. J Dent Res. 2007;86(4):306-19.

6. Menezes $\mathrm{R}$ et al. The potential role of suppressors of cytokine signaling in the attenuation of inflammatory reaction and alveolar bone loss associated with apical periodontitis. J Endod. 2008;34(12):1480-4.

7. Mahanonda R, Pichyangkul S. Toll-like receptors and their role in periodontal health and disease. Periodontol 2000. 2007;43:41-55.

8. Gelani $\mathrm{V}$ et al. The role of toll-like receptor 2 in the recognition of Aggregatibacter actinomycetemcomitans. J Periodontol. 2009;80(12):2010-9.

9. Hajishengallis $\mathrm{G}$ et al. Subversion of innate immunity by periodontopathic bacteria via exploitation of complement receptor-3. Adv Exp Med Biol. 2008;632:203-19.

10. Hajishengallis $\mathrm{G}$ et al. Pathogenic microbes and community service through manipulation of innate immunity. Adv Exp Med Biol. 2012;946:69-85.

11. Onishi S et al. Toll-like receptor 2-mediated interleukin-8 expression in gingival epithelial cells by the Tannerella forsythia leucinerich repeat protein BspA. Infect Immun. 2008;76(1):198-205.

12. Offenbacher S, Barros SP, Beck JD. Rethinking periodontal inflammation. J Periodontol. 2008;79(8 Suppl):1577-84.

13. Eskan MA et al. Interleukin-1beta modulates proinflammatory cytokine production in human epithelial cells. Infect Immun. 2008;76(5):2080-9.

14. Dommisch $\mathrm{H}$ et al. Antimicrobial responses of primary gingival cells to Porphyromonas gingivalis. J Clin Periodontol. 2012;39(10): 913-22.
15. Yoshimura T et al. Purification of a human monocyte-derived neutrophil chemotactic factor that has peptide sequence similarity to other host defense cytokines. Proc Natl Acad Sci U S A. 1987;84(24):9233-7.

16. Scott DA, Krauss J. Neutrophils in periodontal inflammation. Front Oral Biol. 2012;15:56-83.

17. Tsai CC, Ho YP, Chen CC. Levels of interleukin-1 beta and interleukin- 8 in gingival crevicular fluids in adult periodontitis. $\mathrm{J}$ Periodontol. 1995;66(10):852-9.

18. Ertugrul AS et al. Comparison of CCL28, interleukin-8, interleukin-1beta and tumor necrosis factor-alpha in subjects with gingivitis, chronic periodontitis and generalized aggressive periodontitis. J Periodontal Res. 2013;48(1):44-51.

19. Gamonal $\mathrm{J}$ et al. Characterization of cellular infiltrate, detection of chemokine receptor CCR5 and interleukin-8 and RANTES chemokines in adult periodontitis. J Periodontal Res. 2001;36(3): 194-203.

20. Gamonal $\mathrm{J}$ et al. Levels of interleukin-1 beta, -8 , and -10 and RANTES in gingival crevicular fluid and cell populations in adult periodontitis patients and the effect of periodontal treatment. J Periodontol. 2000;71(10):1535-45.

21. Garlet GP et al. Patterns of chemokines and chemokine receptors expression in different forms of human periodontal disease. $\mathrm{J}$ Periodontal Res. 2003;38(2):210-7.

22. Pradeep AR, Daisy H, Hadge P. Serum levels of monocyte chemoattractant protein-1 in periodontal health and disease. Cytokine. 2009;47(2):77-81.

23. Gupta M, Chaturvedi R, Jain A. Role of monocyte chemoattractant protein-1 (MCP-1) as an immune-diagnostic biomarker in the pathogenesis of chronic periodontal disease. Cytokine. 2013;61(3):892-7.

24. Anil $\mathrm{S}$ et al. Increased levels of serum and gingival crevicular fluid monocyte chemoattractant protein-1 in smokers with periodontitis. J Periodontol. 2013;84(9):e23-8.

25. de Brito LC et al. T-lymphocyte and cytokine expression in human inflammatory periapical lesions. J Endod. 2012;38(4):481-5.

26. Gazivoda D et al. Production of proinflammatory and immunoregulatory cytokines by inflammatory cells from periapical lesions in culture. J Oral Pathol Med. 2009;38(7):605-11.

27. Graves DT et al. Interleukin-1 and tumor necrosis factor antagonists inhibit the progression of inflammatory cell infiltration toward alveolar bone in experimental periodontitis. J Periodontol. 1998;69(12):1419-25.

28. Garlet GP et al. The dual role of p55 tumour necrosis factor-alpha receptor in Actinobacillus actinomycetemcomitans-induced experimental periodontitis: host protection and tissue destruction. Clin Exp Immunol. 2007;147(1):128-38.

29. Fukada SY et al. iNOS-derived nitric oxide modulates infectionstimulated bone loss. J Dent Res. 2008;87(12):1155-9.

30. Garlet GP et al. Expression of suppressors of cytokine signaling in diseased periodontal tissues: a stop signal for disease progression? J Periodontal Res. 2006;41(6):580-4.

31. Cardoso $\mathrm{CR}$ et al. Characterization of $\mathrm{CD} 4+\mathrm{CD} 25+$ natural regulatory $\mathrm{T}$ cells in the inflammatory infiltrate of human chronic periodontitis. J Leukoc Biol. 2008;84(1):311-8.

32. Mosmann TR et al. Two types of murine helper T cell clone. I. Definition according to profiles of lymphokine activities and secreted proteins. J Immunol. 1986;136(7):2348-57.

33. Romagnani S. Human TH1 and TH2 subsets: doubt no more. Immunol Today. 1991;12(8):256-7.

34. Szabo SJ et al. A novel transcription factor, T-bet, directs Th1 lineage commitment. Cell. 2000;100(6):655-69.

35. Suzuki Y et al. Interferon-gamma: the major mediator of resistance against Toxoplasma gondii. Science. 1988;240(4851):516-8.

36. Suzuki Y, Remington JS. Dual regulation of resistance against Toxoplasma gondii infection by Lyt-2+ and Lyt-1+, L3T4+ T cells in mice. J Immunol. 1988;140(11):3943-6. 
37. Mosmann TR, Coffman RL. TH1 and TH2 cells: different patterns of lymphokine secretion lead to different functional properties. Annu Rev Immunol. 1989;7:145-73.

38. Zhu J, Paul WE. CD4 T cells: fates, functions, and faults. Blood. 2008;112(5):1557-69.

39. Takeichi $\mathrm{O}$ et al. Cytokine profiles of T-lymphocytes from gingival tissues with pathological pocketing. J Dent Res. 2000;79(8):1548-55.

40. Salvi GE et al. Inflammatory mediators of the terminal dentition in adult and early onset periodontitis. J Periodontal Res. 1998;33(4): 212-25.

41. Vernal $\mathrm{R}$ et al. High expression levels of receptor activator of nuclear factor-kappa B ligand associated with human chronic periodontitis are mainly secreted by CD4 $+\mathrm{T}$ lymphocytes. J Periodontol. 2006;77(10):1772-80.

42. Silva $\mathrm{N}$ et al. Characterization of progressive periodontal lesions in chronic periodontitis patients: levels of chemokines, cytokines, matrix metalloproteinase-13, periodontal pathogens and inflammatory cells. J Clin Periodontol. 2008;35(3):206-14.

43. Vernal $\mathrm{R}$ et al. RANKL in human periapical granuloma: possible involvement in periapical bone destruction. Oral Dis. 2006;12(3): 283-9.

44. Garlet GP et al. The essential role of IFN-gamma in the control of lethal Aggregatibacter actinomycetemcomitans infection in mice. Microbes Infect. 2008;10(5):489-96.

45. Wassenaar A et al. Cloning, characterization, and antigen specificity of T-lymphocyte subsets extracted from gingival tissue of chronic adult periodontitis patients. Infect Immun. 1995;63(6):2147-53.

46. Page RC, Schroeder HE. Pathogenesis of inflammatory periodontal disease. A summary of current work. Lab Invest. 1976;34(3):235-49.

47. Seymour GJ, Cole KL, Powell RN. Analysis of lymphocyte populations extracted from chronically inflamed human periodontal tissues. II. Blastogenic response. J Periodontal Res. 1985;20(6): 571-9.

48. Seymour GJ. Possible mechanisms involved in the immunoregulation of chronic inflammatory periodontal disease. J Dent Res. 1987;66(1):2-9.

49. Gemmell E, Yamazaki K, Seymour GJ. The role of T cells in periodontal disease: homeostasis and autoimmunity. Periodontol 2000. 2007;43:14-40.

50. Gaffen SL, Hajishengallis G. A new inflammatory cytokine on the block: re-thinking periodontal disease and the Th1/Th2 paradigm in the context of Th17 cells and IL-17. J Dent Res. 2008;87(9):817-28.

51. Preshaw PM, Taylor JJ. How has research into cytokine interactions and their role in driving immune responses impacted our understanding of periodontitis? J Clin Periodontol. 2011;38 Suppl 11:6084.

52. Cua DJ et al. Interleukin-23 rather than interleukin-12 is the critical cytokine for autoimmune inflammation of the brain. Nature. 2003;421(6924):744-8

53. Ivanov II et al. The orphan nuclear receptor RORgammat directs the differentiation program of proinflammatory IL-17+ T helper cells. Cell. 2006;126(6):1121-33.

54. Vernal $\mathrm{R}$ et al. Levels of interleukin-17 in gingival crevicular fluid and in supernatants of cellular cultures of gingival tissue from patients with chronic periodontitis. J Clin Periodontol. 2005;32(4):383-9.

55. Yang $\mathrm{S}$ et al. Imbalance of interleukin-17+ T-cell and foxp3+ regulatory T-cell dynamics in rat periapical lesions. J Endod. 2014;40(1):56-62.

56. Cardoso CR et al. Evidence of the presence of T helper type 17 cells in chronic lesions of human periodontal disease. Oral Microbiol Immunol. 2009;24(1):1-6.

57. Beklen A et al. MMPs, IL-1, and TNF are regulated by IL-17 in periodontitis. J Dent Res. 2007;86(4):347-51.

58. Di Benedetto A et al. Periodontal disease: linking the primary inflammation to bone loss. Clin Dev Immunol. 2013;2013:503754.
59. Park $\mathrm{H}$ et al. A distinct lineage of CD4 T cells regulates tissue inflammation by producing interleukin 17 . Nat Immunol. 2005;6(11):1133-41.

60. Langrish CL et al. IL-23 drives a pathogenic T cell population that induces autoimmune inflammation. J Exp Med. 2005;201(2):23340.

61. Dutzan $\mathrm{N}$ et al. Over-expression of forkhead box P3 and its association with receptor activator of nuclear factor-kappa B ligand, interleukin (IL) -17, IL-10 and transforming growth factor-beta during the progression of chronic periodontitis. J Clin Periodontol. 2009;36(5):396-403.

62. Dutzan $\mathrm{N}$ et al. Levels of interferon-gamma and transcription factor T-bet in progressive periodontal lesions in patients with chronic periodontitis. J Periodontol. 2009;80(2):290-6.

63. Cottrez $\mathrm{F}$ et al. T regulatory cells 1 inhibit a Th2-specific response in vivo. J Immunol. 2000;165(9):4848-53.

64. Garlet GP et al. Regulatory T cells attenuate experimental periodontitis progression in mice. J Clin Periodontol. 2010;37(7):591-600.

65. Walker KF et al. Cytokine expression in periapical granulation tissue as assessed by immunohistochemistry. Eur J Oral Sci. 2000;108(3):195-201.

66. Andrade AL et al. Immunoexpression of interleukin 17, transforming growth factor beta1, and forkhead box P3 in periapical granulomas, radicular cysts, and residual radicular cysts. J Endod. 2013;39(8):990-4.

67.• Glowacki AJ et al. Prevention of inflammation-mediated bone loss in murine and canine periodontal disease via recruitment of regulatory lymphocytes. Proc Natl Acad Sci U S A. 2013;110(46):1852530. The article describes a method to harness endogenous Tregs and recruit them to specific sites of aberrant inflammation, locally halting tissue destruction and establishing a regenerative milieu.

68. Jager A, Kuchroo VK. Effector and regulatory T-cell subsets in autoimmunity and tissue inflammation. Scand J Immunol. 2010;72(3):173-84.

69. Elyaman W et al. IL-9 induces differentiation of TH17 cells and enhances function of FoxP3 + natural regulatory T cells. Proc Natl Acad Sci U S A. 2009;106(31):12885-90.

70. Schlapbach C et al. Human TH9 Cells Are Skin-Tropic and Have Autocrine and Paracrine Proinflammatory Capacity. Sci Transl Med. 2014;6(219):219ra8.

71. Manni M, Robinson KR, Alcorn JF. A tale of two cytokines: IL-17 and IL-22 in asthma and infection. Expert Rev Respir Med. 2014;8(1):25-42.

72. Alghasham A, Rasheed Z. Therapeutic targets for rheumatoid arthritis: Progress and promises. Autoimmunity, 2014

73. Kim KW et al. Interleukin-22 promotes osteoclastogenesis in rheumatoid arthritis through induction of RANKL in human synovial fibroblasts. Arthritis Rheum. 2012;64(4):1015-23.

74.• Aranha AM et al. Evidence supporting a protective role for th9 and th22 cytokines in human and experimental periapical lesions. J Endod. 2013;39(1):83-7. The article describes the contribution of Th9 and Th22 lymphocytes in the pathogenesis of human and experimental periapical lesions.

75. MacLennan IC. Germinal centers. Annu Rev Immunol. 1994;12: 117-39.

76. Tangye SG et al. The good, the bad and the ugly - TFH cells in human health and disease. Nat Rev Immunol. 2013;13(6):412-26.

77. Dutzan $\mathrm{N}$ et al. Interleukin-21 expression and its association with proinflammatory cytokines in untreated chronic periodontitis patients. J Periodontol. 2012;83(7):948-54.

78. Napimoga MH et al. Possible involvement of IL-21 and IL-10 on salivary IgA levels in chronic periodontitis subjects. Scand J Immunol. 2011;74(6):596-602.

79. Kwok SK et al. Interleukin-21 promotes osteoclastogenesis in humans with rheumatoid arthritis and in mice with collageninduced arthritis. Arthritis Rheum. 2012;64(3):740-51. 
80. Zhao L et al. Effect of non-surgical periodontal therapy on the levels of Th17/Th1/Th2 cytokines and their transcription factors in Chinese chronic periodontitis patients. J Clin Periodontol. 2011;38(6):509-16.

81. Fabrizi M, et al. IL-21 is a major negative regulator of IRF4dependent lipolysis affecting Tregs in adipose tissue and systemic insulin sensitivity. Diabetes, 2014.

82. McGeachy MJ, Cua DJ. Th17 cell differentiation: the long and winding road. Immunity. 2008;28(4):445-53.

83.• O'Shea JJ, Paul WE. Mechanisms underlying lineage commitment and plasticity of helper CD4+ T cells. Science. 2010;327(5969): 1098-102. The article reviews the recent advancements in CD4+ $T$ helper cells linage commitment and plasticity research, focusing in the therapeutic implications of the new findings.
84. Saraiva M, O'Garra A. The regulation of IL-10 production by immune cells. Nat Rev Immunol. 2010;10(3):170-81.

85. Schmitt E, Klein M, Bopp T. Th9 cells, new players in adaptive immunity. Trends Immunol, 2013.

86. Veldhoen $\mathrm{M}$ et al. Transforming growth factor-beta 'reprograms' the differentiation of $\mathrm{T}$ helper 2 cells and promotes an interleukin 9producing subset. Nat Immunol. 2008;9(12):1341-6.

87. Lochner $M$ et al. In vivo equilibrium of proinflammatory IL-17+ and regulatory IL-10+ Foxp3+ RORgamma t+T cells. J Exp Med. 2008;205(6):1381-93.

88. Lee YK et al. Developmental plasticity of Th17 and Treg cells. Curr Opin Immunol. 2009;21(3):274-80.

89. Gardner M. Mathematical Games: The fantastic combinations of John Conway's new solitaire game "life". Sci Am. 1970;223(Oct):120-3. 